\title{
Research on Application of an Optimized Method though Self-learning Fuzzy Neural Network for Ore Slurry Concentration in Flotation Process
}

\author{
Xiaoqing Liu*, Liumin Luo and Jin Li
}

School of Physics and Mechanical \& Electrical Engineering, Zhoukou Normal University, Zhoukou, Henan, 466001, China

\begin{abstract}
Feed concentration directly affects the recovery of mineral resources in flotation process, which is an important method of separating fine-grained mineral. Due to complicated process and mechanism of thickener, the control effect is poor with the traditional control method under the condition of time-varying process parameters. Fuzzy control and BP neural network are combined with together in this paper, then we propose a optimization method though self-learning fuzzy neural network, and solved the problem of optimal controlling for the system with variable parameters. Applied to the production process of thickener, the result of instance simulation shows that it can elegantly solve the problem of controlling the ore concentration.
\end{abstract}

Keywords: BP neural network, Control of ore slurry concentration, Flotation process, Fuzzy control, Parameter optimization.

\section{INTRODUCTION}

Mineral resources play a vital role in the economic development process. Beneficiation is an important part in the processing of mineral resources, so the level of beneficiation will directly affect the recovery of mineral resource [1]. For this reason, what needs to be resolved urgently in beneficiation industry is that, how to choose an effective method to control the process of dressing. Regardless of gravity separation, electrostatic separation, magnetic separation, or flotation, beneficiation process will be limited by granularity [2]. This will provide too thick to sort, while too thin to recycle [3]. Flotation is an important method for sorting particulate mineral [4], based on differences in physical and chemical properties on the surface of the mineral. By adjusting the feeding capacity, ore concentration, dose, inflating volume, liquid level and other parameters [5], the flotation pulp is separated into concentrate and tailings with qualified grade, to achieve effective sorting [6]. Among these parameters, the size of the ore concentration plays a vital role in the production process of flotation. If not controlled in a suitable range, the accidents such as froth overflow and no foam will occur $[7,8]$, leading to that the production process can not be carried out smoothly and stably. Therefore, the PID controller is used to adjust the ore concentration. Due to the complexity of the movement of pulp, with nonlinear and time-varying characteristics, it is difficult to establish a precise mathematical model for the ore concentration. Though the traditional PID parameter tuning methods are convenient and practical, the PID parameters can not be adjusted in real-time according to changing of the control object, hence; it is difficult to obtain better control effect. To compensate for the lack of the above control technology, BP neural network and fuzzy control are introduced in this paper. With this method, online optimization parameters can be obtained, thereby control accuracy of the ore concentration will be raised.

\section{DESCRIPTION OF FLOTATION PROCESS}

Sorting capacity of single flotation devices is limited; therefore it is usually difficult to meet the technical requirements of the process. Then we consider using multi devices in series and parallel, as shown in Fig. (1).

Dewatering operation is conducted, when the pulp from the procedure before is fed to the thickener [9]. The ore slurry with appropriate concentration enters into the feed box through the slurry pumps, then go to the agitator of the flotation workshop for full mashing. In the meantime, add the appropriate flotation agent to the mixing bucket, making full interaction between agent and pulp, to mineralize the mineral at the extreme. Pulp after interaction flows through the overflow port, then enter into the rougher flotation column with a relatively low height. After the initial flotation, the concentrates are collected directly into the concentrate slot, and flotation tailings were transported to the first scavenging flotation column to be scavenged. Then the concentrates from the first scavenging enter into the middling tank as middlings, while the tailings go to the second scavenging flotation column to be scavenged once again. The concentrates from the second scavenging are discharged into the same middling tank as meddling. Finally, the slurry in the middling tank is pumped back to the thickener, while the tailings from the second scavenging are taken as the final tailings. 


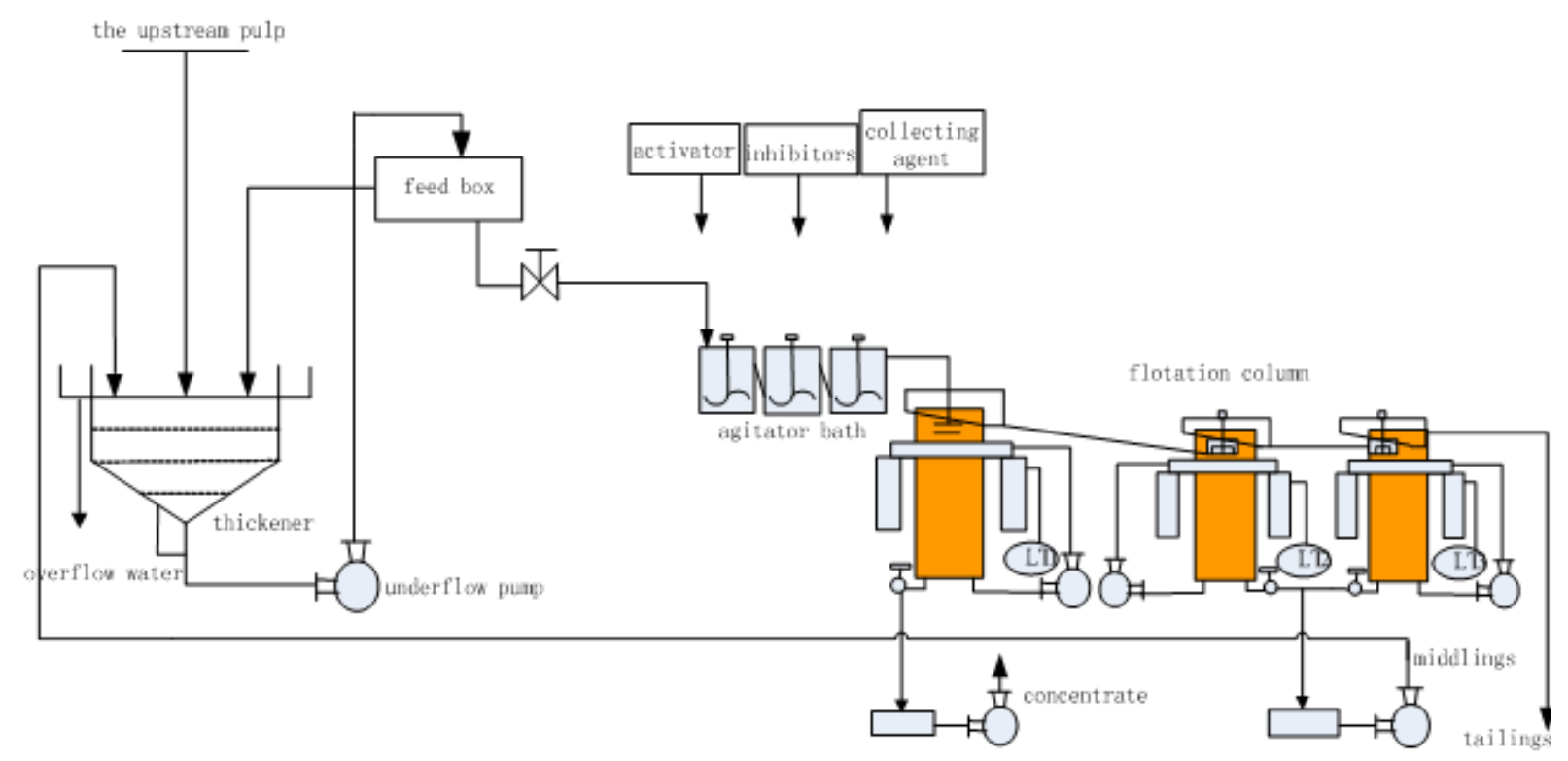

Fig. (1). Flowchart of the floatation process.

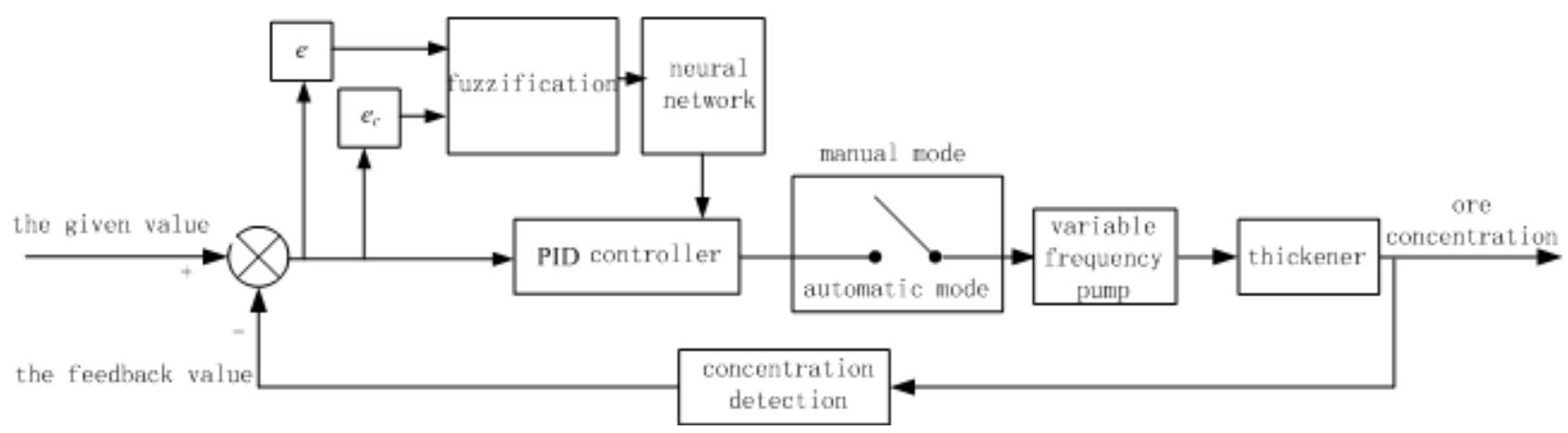

Fig. (2). Control block diagram of optimizing PID parameters with fuzzy neural network.

\section{OPTIMIZATION OF PID PARAMETERS WITH FUZZY INFERENCE AND NEURAL NETWORK}

PID controller, with the advantages of simple structure, high reliability, is widely used in the industry; however its adaptability is relatively poor. Fuzzy inference is based on artificial expertise and knowledge rule, and neural network possesses the abilities of learning and cognizing. For delay, time varying, and nonlinear system, they have good control capability. Nevertheless, due to the lack of integral part, there will be steady-state error usually. So we will combine fuzzy control, neural network, and PID controller, to achieve good robustness, fast response and high control precision. The main idea is that, modify PID parameters online through fuzzy rule and neural network, according to the error $e$ between the given value and the feedback value of ore concentration, and the rate of changing of the error $e_{c}$, as depicted in Fig. (2).

In this control loop, set point of concentration is the input, and its detection value is the output. The error $e$ between the input and the output, and the rate of changing of the error $e_{c}$, are taken as the input of fuzzy neural network. Then frequency of the frequency converter depends on the output of the PID controller that is control action. Besides, we set both automatic and manual adjustment mode. In automatic mode, the frequency of the frequency converter is adjusted by fuzzy neural network and PID controller, while it is simply set by hand in manual mode. The concentration of underflow slurry from the thickener depends on residence time of pulp in the compression zone. So we can get more stable underflow concentration by adjusting the frequency of the frequency converter, which determines speed of the underflow pump, and then determines the residence time.

\subsection{Fuzzy Set and Membership Function}

The method in this paper uses the form of two inputs and three outputs, with the error $e$ and the rate of changing of the error $e_{c}$ as inputs, and $\Delta K_{P}, \Delta K_{I}, \Delta K_{D}$ as outputs. Take the domain of discourse for $[-5,5]$, and obscure the inputs and 


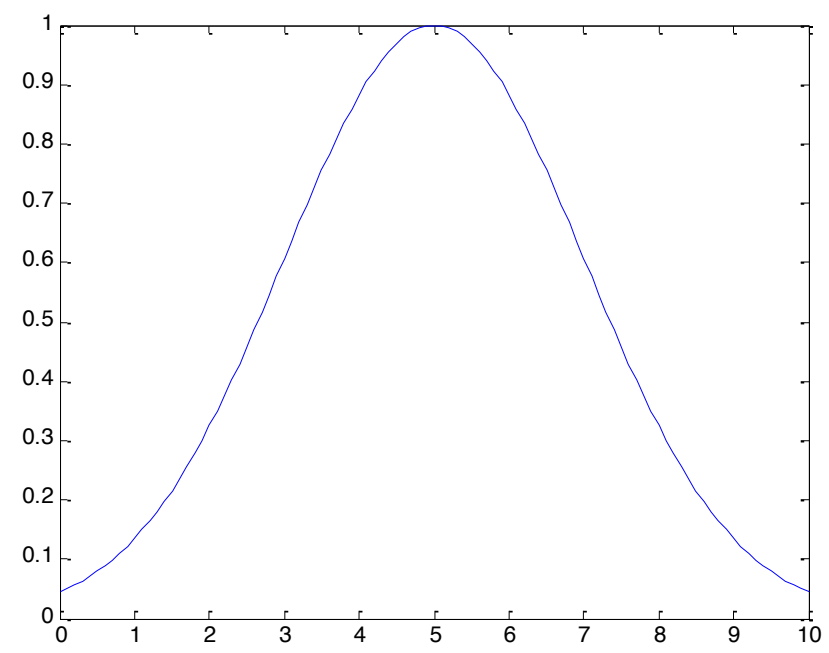

Fig. (3). Gaussian membership function.

outputs. In the process of obscuring, $e, e_{c}, \Delta K_{P}, \Delta K_{I}, \Delta K_{D}$ correspond to fuzzy subset $\{\mathrm{NB}, \mathrm{NM}, \mathrm{NS}, \mathrm{Z}, \mathrm{PS}, \mathrm{PM}, \mathrm{PB}\}$, which represent Negative Big, Negative Medium, Negative Small, Zero, Positive Small, Positive Medium, Positive Big respectively. Use Gaussian membership function for $e$ and $e_{c}$, and sigmoid membership function for $\Delta K_{P}, \Delta K_{I}$, and $\Delta$ $K_{D}$. These two kinds of membership functions have good smoothness, there are not zero in graphics, and have clear physical meaning, so they are the most commonly used to describe uncertainty. The expressions of gaussian membership function [10] is represented as shown below:

$y=e^{\frac{-(x-c)^{2}}{2 \sigma^{2}}}$

Thereinto, $c$ determines center of the function, and $\sigma$ determines width of the function curve, as shown in Fig. (3).

Sigmoid membership function is indicated as follows:

$y=\frac{1}{1+e^{-a(x-c)}}$

It applies to membership function with the modifying language value of "very", "is not". As shown in Fig. (4), when $\mathrm{a}$ is positive, the curve of sigmoid membership function curves to the right, while it curves to the left when a is negative.

\subsection{Rule of Fuzzy Inference}

The following several ways are the commonly used methods to generate fuzzy control rules:

(1) Generate control rules according to the experience of the experts or the knowledge of process control;

(2) Generate control rules according to fuzzy model of the process;
(3) Generate control rules according to systematic observation and measurement of the manual operation.

Based on vast experience knowledge of operator and data analysis in the flotation process, we establish fuzzy inference rules concerning the ore concentration. The specific implementation process is as follows:

(1) If $e$ is very big, we should make it decrease rapidly, and then large $K_{P}$ should be taken. Meanwhile, in order to avoid differential saturation and appearing overshoot, $K_{I}, K_{D}$ should be small.

(2) If $e$ and $e_{c}$ are medium, to gain a smaller overshoot in the system, $K_{P}$ should be smaller and $K_{I}, K_{D}$ should be modest.

(3) If $e$ is small, in order to improve the dynamic performance and steady-state performance of the system, $K_{P}$ and $K_{I}$ should be small while $K_{D}$ should be medium. If $e_{c}$ is largish, $K_{P}$ should increase appropriately.

In accordance with the above experience, the corresponding reasoning rule is listed in Table $\mathbf{1}$.

Then PID parameters are adjusted by the formula:

$$
\begin{aligned}
& K_{P}=\Delta K_{P}+K_{P 0} \\
& K_{I}=\Delta K_{I}+K_{I 0} \\
& K_{D}=\Delta K_{D}+K_{D 0}
\end{aligned}
$$

Where $\Delta K_{P}, \Delta K_{I}, \Delta K_{D}$ are the outcome of fuzzy reasoning, that is parameter adjustment; $K_{P 0}, K_{I 0}, K_{D 0}$ are the initial value of the parameters.

\subsection{Design of BP Neural Network}

According to input information, BP neural network calculates the output value of each unit layer by layer through the hidden layer, then reversely calculates the error of each 


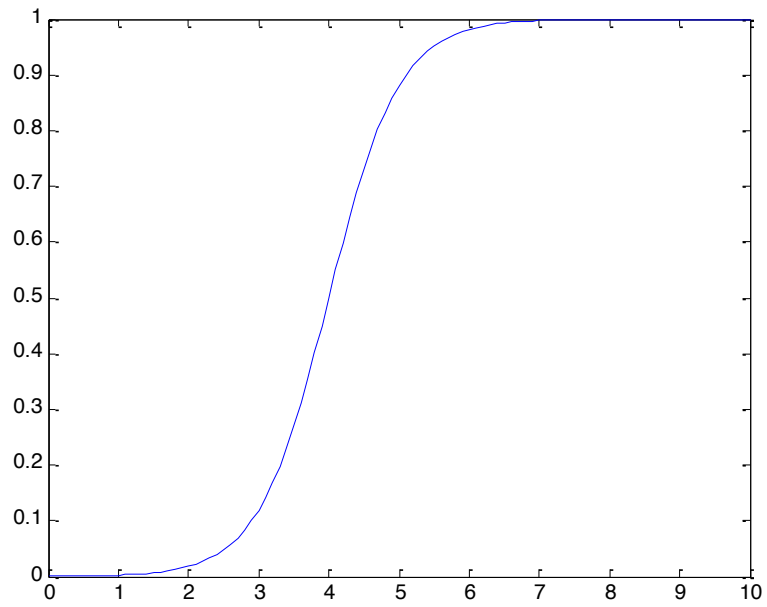

(a) $a>0$

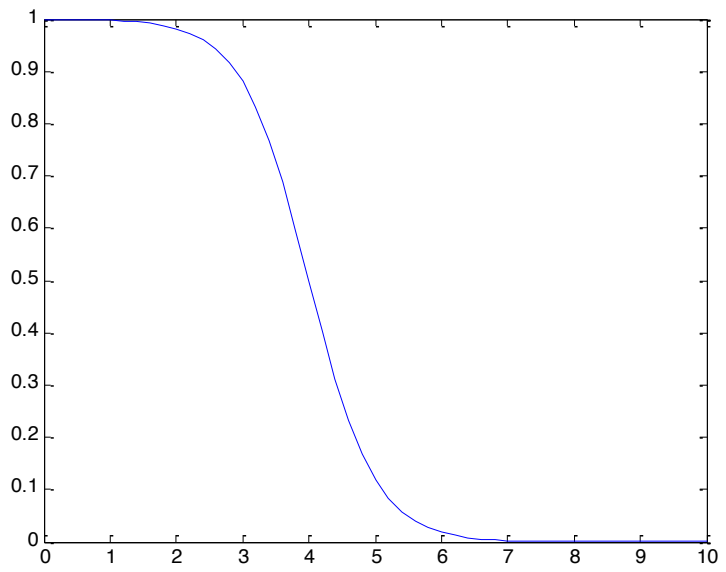

(b) a $<0$

Fig. (4). Sigmoid membership function.

Table 1. Reasoning rule.

\begin{tabular}{|c|c|c|c|c|c|c|c|}
\hline $\mathbf{C u}_{\mathrm{c}}$ & NB & NM & NS & $\mathbf{Z}$ & PS & PM & PB \\
\hline NB & PB & PB & $\mathrm{PB}$ & $\mathrm{PM}$ & $\mathrm{PM}$ & PS & Z \\
\hline $\mathrm{NM}$ & $\mathrm{PB}$ & $\mathrm{PB}$ & $\mathrm{PM}$ & $\mathrm{PM}$ & PS & Z & NS \\
\hline NS & PB & $\mathrm{PM}$ & PS & PS & Z & Z & $\mathrm{NM}$ \\
\hline Z & $\mathrm{PM}$ & PS & PS & Z & Z & NS & $\mathrm{NM}$ \\
\hline PS & PS & Z & Z & Z & NS & NM & NB \\
\hline $\mathrm{PM}$ & $\mathrm{Z}$ & Z & NS & NS & NM & NB & NB \\
\hline PB & $\mathrm{Z}$ & NS & NM & NM & NB & NB & NB \\
\hline
\end{tabular}




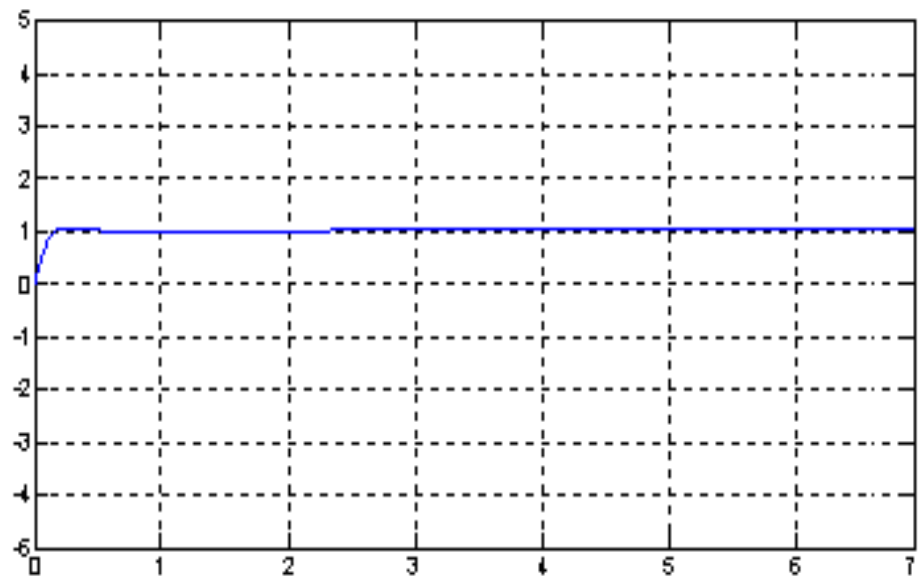

Fig. (5). The step response curve for slurry consistency.

unit in the hidden layer based on output error [11], finally corrects weights of layers before. Specific steps are as follows:

As input layer, the first layer with two neurons [12] sends the error $e$ and the rate of changing of the error $e_{c}$ to neurons of the next layer respectively. That is, $\mathrm{x}_{1}=E(\mathrm{t}), \mathrm{x}_{2}=E c(\mathrm{t})$.

In the hidden layer, suppose there is $n$ neurons in the $q$ th layer, and connection weight from input to the $i$-th neuron in the $q$-th layer is $\omega_{i j}{ }^{q}$, then the transformation relation between input and output of the multilayer perceptron network is that,

$$
S_{i}^{q}=\sum_{j=0}^{n_{q-1}} \omega_{i j}{ }^{q} x_{j}^{q-1}
$$

Where $\omega_{i 0}{ }^{q}=-1 ; x_{0}^{q-1}=\theta_{i}^{q} ; x_{i}^{q-1}=\frac{1}{1+e^{-\mu s_{i}^{q}}}$

$$
i=1,2, \ldots, n_{q} ; j=1,2, \ldots, n_{q-1} ; q=1,2, \ldots, Q \text {. }
$$

For BP neural network, take the cost function of the fitting error function as

$$
\begin{aligned}
& E=\frac{1}{2} \sum_{p=1}^{P} \sum_{i=1}^{n_{Q}}\left(d_{p i}-x_{p i}{ }^{Q}\right)=\sum_{p=1}^{P} E_{p} \\
& E_{p}=\sum_{i=1}^{n_{Q}}\left(d_{p i}-x_{p i}{ }^{Q}\right)^{2}
\end{aligned}
$$

Then, for the (q-1)-th layer,

$$
\begin{aligned}
\partial E_{p} / \partial \omega_{i j}{ }^{Q-1} & =\frac{\partial E_{p}}{\partial x_{p i}{ }^{Q-1}} \frac{\partial x_{p i}{ }^{Q-1}}{\partial \omega_{i j}{ }^{Q-1}} \\
& =\left(\sum_{k=1}^{n_{Q}}-\delta_{p k}{ }^{Q} \omega_{k i}{ }^{Q}\right) f^{\prime}\left(S_{p i}{ }^{Q-1}\right) x_{p j}{ }^{Q-2} \\
& =-\delta_{p i}{ }^{Q-1} x_{p j}{ }^{Q-2}
\end{aligned}
$$

$\delta_{p i}{ }^{Q-1}=-\frac{\partial E_{p}}{\partial x_{p i}{ }^{Q-1}}=\left(\sum_{k=1}^{n_{Q}} \delta_{p k}{ }^{Q} \omega_{k i}{ }^{Q}\right) f^{\prime}\left(S_{p i}{ }^{q-1}\right)$

Take $\mathrm{f}($.$) as sigmoid function here, its derivative can be$ obtained by

$$
\begin{gathered}
x_{p i}{ }^{q}=f\left(S_{p i}{ }^{q}\right)=\frac{1}{1+e^{-\mu S_{p i}{ }^{q}}} \\
f^{\prime}\left(S_{p i}{ }^{q}\right)=\mu x_{p i}{ }^{q}\left(1-x_{p i}{ }^{q}\right)
\end{gathered}
$$

At last, learning algorithm of BP neural network can be summarized as

$$
\begin{gathered}
W_{i j}^{q}(k+1)=w_{i j}^{q}(k)+\alpha D_{i j}^{q}(k) \\
D_{i j}^{q}=\sum_{p=1}^{P} \delta_{p i}{ }^{q} x_{p j}{ }^{q-1}
\end{gathered}
$$

\section{SYSTEM SIMULATION}

In the process of thickener production, the mathematic model of the relation between frequency of underflow pump and density of underflow pulp can be obtained through the test method commonly used in engineering. That is,

$W(s)=\frac{-3.6}{20 s+1} e^{-5 s}$

Optimize the PID parameters using the method aforementioned, and conduct simulation. The control effect is shown in Fig. (5).

As seen in Fig. (5), when we give a step signal to ore concentration, the output response is fast and stable, with overshoot within the scope of the permit. It shows that both dynamic and static characteristics can achieve the ideal effect. 


\section{CONCLUSION}

In order to control ore concentration in the flotation process, PID control, fuzzy control, BP neural network are combined with together in this paper. Through self-learning and fuzzy reasoning, the parameters are optimized. The result indicates that the control method is effective, and has fine self-adaptive capacity.

\section{CONFLICT OF INTEREST}

The authors confirm that this article content has no conflict of interest.

\section{ACKNOWLEDGEMENTS}

The research of this paper has been sponsored by Henan Provincial Research Foundation for Science and Technological Breakthroughs, China (Grant No. 132102210179, 142102210597), Natural Science Foundation of Henan Educational Committee, China (Grant No.2011B510021).

\section{REFERENCES}

[1] W. Gui, C. Yang, D. Xu, M. Lu, and Y. Xie, "Machine-visionbased online measuring and controlling technologies for mineral flotation-a review," Acta Automatica Sinica, vol. 39, pp. 18791888, 2013.
[2] P. Zhou, and J.L. Ding, "An intelligent approach for supervisory control of grinding product particle size," In: The $46^{\text {th }}$ IEEE Conference on Decision and Control, 2007, no. 5, pp. 1856- 1861.

[3] Z. Geng, and T. Chai, "Intelligently optimal index setting for flotation process by CBR," Journal of Northeastern University, vol. 29, no. 6, pp. 761-764, 2008.

[4] J.J. Liu, J.F. Macgrego, and C. Duchesne, "Flotation froth monitoring using multire solutional multivariate image analysis," Minerals Engineering, vol. 33, no. 5, pp. 65-76, 2005.

[5] W. Gui, Z. Chen, M. He, and C. Yang, "Soft sensor of sulfur flotation froth level based on relevance vector machine," Control Engineering of China, vol. 21, no. 1, pp. 1-4, 2014.

[6] H. Li, T. Chai, and Y. Heng, "Automatic control system of flotation processes," Control Engineering of China, vol. 20, no. 5, pp. 796-799, 2013.

[7] J. Z. Lu, "Challenging control problems and emerging technologies in enterprise optimization," Control Engineering Practice, vol. 29, no. 12, pp. 847- 858, 2003.

[8] H. Li, X. Zheng, and T. Chai, "Hybrid intelligent optimal control in flotation processes," Journal of Northeastern University, vol. 33, no. 1, pp. 1-5, 2012.

[9] Y. Zhang, J. Wang, and W. Wang, "Application of expert control method to flotation process," Control and Decision, vol. 19, no. 11, pp. 1271-1274, 2004.

[10] J. Wang, Q. Liu, and Q. Dang, "Nonlinear membership function established by single- shot clustering method," Journal of Zhengzhou University (Engineering Science), vol. 33, no. 2, pp. 28-30, 2014.

[11] L. Zong, X. Li, and R. Deng, "Fuzzy PID air volume control based on BP neural network," Journal of Guilin University of Electronic Technology, vol. 34, no. 2, pp. 131-134, 2014.

[12] H. Liu, K. Li, and J. Liu, "Applicationg of fuzzy pid control based on bp neural network on flux control of coal gas for furnace," $\mathrm{Hy}$ drometallurgy of China, vol. 20, no. 2, pp.120-123, 2008.

(C) Liu et al.; Licensee Bentham Open.

This is an open access article licensed under the terms of the Creative Commons Attribution Non-Commercial License (http://creativecommons.org/licenses/by-nc/3.0/) which permits unrestricted, non-commercial use, distribution and reproduction in any medium, provided the work is properly cited. 